\title{
Primary lung sarcoma treated with stereotactic ablative radiotherapy: a case report
}

This article was published in the following Dove Press journal:

OncoTargets and Therapy

4 July 2017

Number of times this article has been viewed

\section{Seung-Gu Yeo}

Department of Radiation Oncology, Soonchunhyang University College of Medicine, Soonchunhyang University

Hospital, Cheonan, Republic of Korea
Abstract: Primary lung sarcoma (PLS) is an extremely rare, very aggressive malignancy. Surgical removal is considered the treatment of choice, and patients who have been given conventional radiotherapy have had inferior outcomes. This study is the first describing a case of PLS treated with stereotactic ablative radiotherapy (SABR), which precisely targets a small tumor with a markedly higher biologically effective dose than conventional radiotherapy. The patient was an 82-year-old man who was diagnosed with primary lung leiomyosarcoma based on radiology, pathology, and immunohistochemical examinations. The PLS was located in the right lower lobe and measured $2.5 \mathrm{~cm}$. No regional nodal or distant organ metastasis was observed. He was inoperable medically. The SABR was performed using volumetric modulated arc therapy and a dose of $56 \mathrm{~Gy}$ in four fractions. Follow-up computed tomography 2 months after SABR revealed a complete tumor response. The toxicity was limited to mild respiratory symptoms. The patient is alive and has had no evidence of disease for 2 years. This study suggests that SABR can be a safe and effective treatment option for PLS.

Keywords: primary lung sarcoma, leiomyosarcoma, stereotactic ablative radiotherapy, stereotactic body radiotherapy, radiation therapy, sarcoma

\section{Introduction}

Primary lung sarcoma (PLS) is a very rare tumor, accounting for $<0.5 \%$ of all lung cancers. ${ }^{1}$ The literature on PLS is limited to small case series. The most common histological type of PLS is leiomyosarcoma. ${ }^{2,3}$ PLS is a very aggressive malignancy; it has a significantly worse 5-year overall survival than extremity soft tissue sarcomas (35\% vs 71\%). ${ }^{4}$ The treatment of PLS is not standardized because of its rarity; however, complete surgical removal reportedly confers the best chance of cure. ${ }^{4}$

Stereotactic ablative radiotherapy (SABR), also known as stereotactic body radiotherapy (RT), is one of the newest and most sophisticated forms of RT. SABR uses high-precision external beam RT to target small tumors with a high radiation dose per fraction in a few treatment courses. 5,6 Compared to conventional RT, SABR substantially improves local control and survival in patients with inoperable early-stage non-small-cell lung cancer (NSCLC). The SABR outcomes for these patients are comparable to those following surgical resection. ${ }^{5}$ In addition, some studies have reported favorable outcomes using SABR in the management of lung metastases from extrapulmonary sarcoma. ${ }^{7-9}$ However, there are no reports on SABR for PLS yet.

This case study presents a patient with PLS who showed a complete tumor response to $\mathrm{SABR}$ and is alive with no disease recurrence or severe toxicity. 


\section{Case report}

An 82-year-old man presented with blood-tinged sputum, dizziness, and a $3 \mathrm{~kg}$ weight loss over 6 months. He had been taking medications for hypertension and diabetes, and had had pulmonary tuberculosis 40 years earlier. He quit smoking 13 years earlier, but had a smoking history of 60 pack-years. Written informed consent has been provided by the patient to have the case details and any accompanying images published.

Chest computed tomography (CT) revealed multiple fluid-containing bullae and a contrast-enhancing $2.5 \mathrm{~cm}$ mass with a lobulated margin in the right lower lobe of the emphysematous lung (Figure 1A). Histologically, tissue from a percutaneous thoracic needle biopsy of this mass showed that it consisted predominantly of spindle-shaped cells with moderate pleomorphism, hemorrhage, and necrosis. The mitotic rate was four figures in 10 high-power fields. Immunohistochemical staining was positive for desmin and smooth muscle actin, and negative for ALK, Pan-CK, CAM5.2, HMB45, Melan-A, P40, and TTF-1. The pathology and immunohistochemistry findings were consistent with leiomyosarcoma. ${ }^{10}$ Whole-body ${ }^{18} \mathrm{~F}$-fluorodeoxyglucose positron emission tomography-CT did not detect any pathologically increased uptake, except above the mass, with a maximum standardized uptake value of 2.24. No specific findings were seen on brain magnetic resonance imaging.

The patient was deemed inoperable medically because of his poor medical condition and old age. The small tumor size and its location at the lung periphery permitted the application of SABR, instead of conventional RT. During the simulation, the patient was immobilized in the supine position with his arms above his head. Respirationcorrelated four-dimensional CT was conducted using a Real-Time Position Management system (Varian Medical Systems; Palo Alto, CA, USA) and a 16-slice CT scanner (Brilliance CT Big Bore, Philips Medical Systems, Cleveland, OH, USA). Each of $10 \mathrm{CT}$ images reflected $10 \%$ of the respiratory cycle. The clinical target volume was delineated on the CT images for each respiratory phase using the lung window setting. The planning target volume was created by adding a $0.5 \mathrm{~cm}$ isotropic setup margin around the internal target volume, which was the sum of the
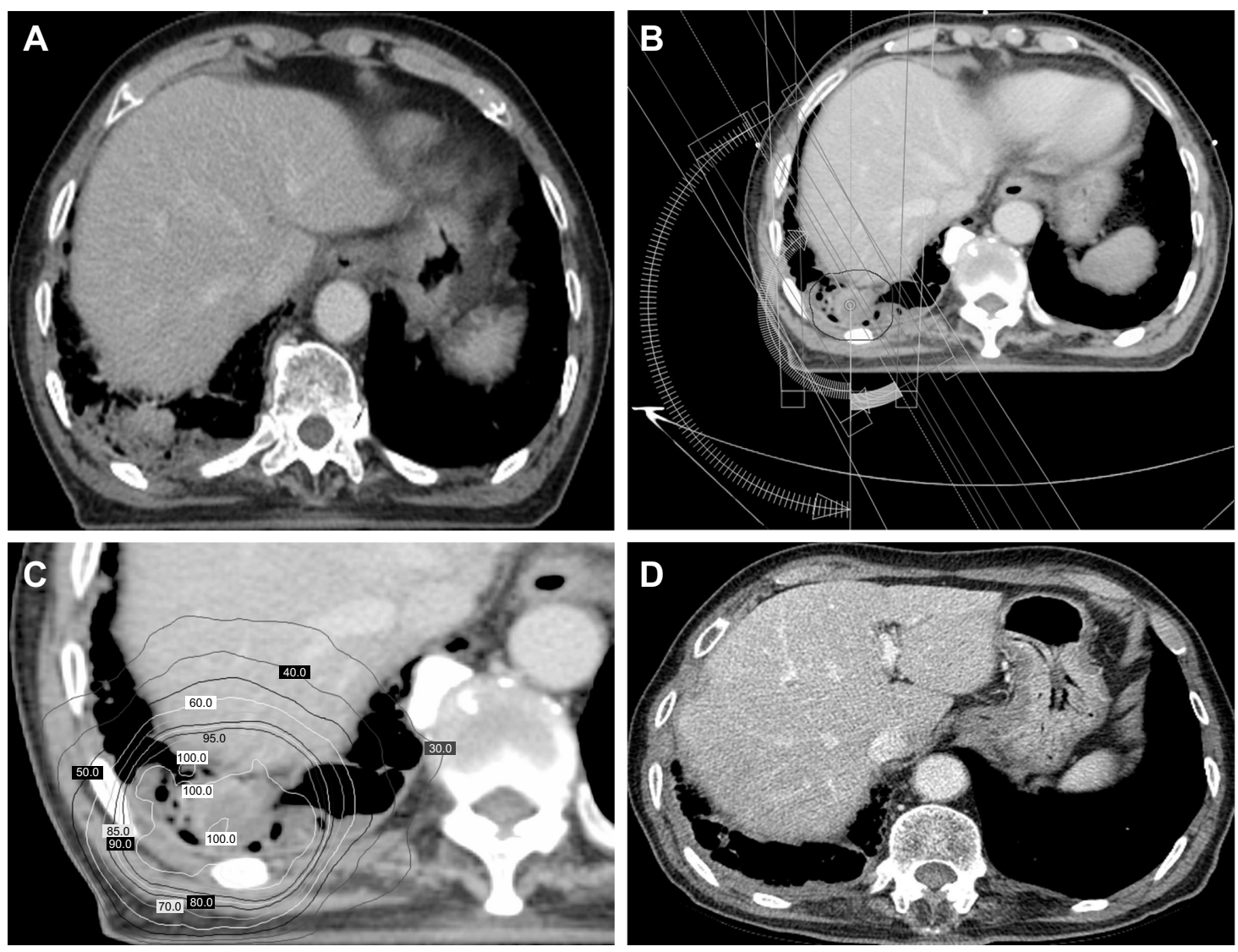

Figure I Pretreatment $2.5 \mathrm{~cm}$ contrast-enhancing mass in the right lower lobe (A). Volumetric modulated arc therapy plan with the planning target volume (B). Dose distribution with isodose lines, from 100\% to $30 \%$ (C). Complete tumor response 2 months after treatment (D). 
clinical target volumes during all 10 respiratory phases. ${ }^{11}$ A volumetric modulated arc therapy plan was created using the Eclipse treatment-planning system (Varian Medical Systems) and $6 \mathrm{MV}$ photons, with inhomogeneity corrections (Figures $1 \mathrm{~B}$ and $\mathrm{C}$ ). ${ }^{5,12}$ The dose fractionation scheme was 56 Gy in four fractions delivered every other day in February 2015. SABR was performed on a Novalis Tx system (Varian Medical Systems and Brainlab, Feldkirchen, Germany).

A complete tumor response to $\mathrm{SABR}$ was observed on chest CT 2 months after the SABR (Figure 1D). Diffuse consolidation with ground glass opacity in the right lower lobe indicated the development of radiation pneumonitis. He developed a productive cough for about 1 month, but this subsided with conservative management. Follow-up chest CT was repeated every 3 or 4 months, and he is alive with no evidence of disease for 2 years.

\section{Discussion}

A retrospective analysis of the Surveillance, Epidemiology, and End Results database showed that the 5-year survival of PLS patients was $41 \%$ after surgery alone $(n=274), 25 \%$ after surgery and adjuvant RT $(\mathrm{n}=52)$, and $7 \%$ for those who underwent RT alone $(\mathrm{n}=39 ; P<0.0001) .{ }^{4}$ However, this RTalone outcome was observed following conventional RT. To the best of the author's knowledge, no SABR outcomes for PLS have been reported. The current study is the first to present a PLS case managed with SABR. The patient showed a complete tumor response to SABR and has shown no evidence of disease for 2 years.

The major reason for poor tumor control with conventional RT in NSCLC has been an insufficient total radiation dose, which is typically $\leq 60$ Gy. ${ }^{5}$ SABR delivers a much higher biological effective dose compared to conventional RT. Up to $60 \mathrm{~Gy}$ is delivered in SABR in as few as three fractions, and this is presumably equal to $150 \mathrm{~Gy}$ in conventional fractionation. ${ }^{13}$ The biologically effective dose of 56 Gy in four fractions used in the present study was $134.4 \mathrm{~Gy}_{10}{ }^{14}$ In addition to this direct killing effect on tumor cells, recent evidence indicates that SABR destroys the tumor vascular bed and thereby deteriorate the intratumoral microenvironment leading to indirect tumor cell death. ${ }^{15}$ SABR has reduced local failure $(<10 \%)$ comparable to the rates following surgery in patients with early-stage NSCLC. ${ }^{5}$ The Radiation Therapy Oncology Group phase II trial reported a 3-year local control rate of $97.6 \% .{ }^{16} \mathrm{SABR}$ is becoming a standard therapeutic option for early-stage NSCLC patients who are medically inoperable or refuse surgery.
For lung metastases from soft tissue sarcoma, SABR results in excellent local control with minimal toxicity. Dhakal et $\mathrm{al}^{7}$ analyzed the SABR outcomes in 15 patients (74 lesions) with pulmonary metastases from soft tissue sarcomas. Leiomyosarcoma, malignant fibrous histiocytoma, and synovial sarcoma were the most common histologies. The preferred dose fractionation was 50 Gy in 5 Gy fractions. The 3-year local control rate was $82 \%$. Five of the seven local failures were in a single patient who was treated with purely palliative intent (30 Gy in 10 fractions). No patient experienced grade $\geq 3$ toxicity. The median survival was 2.1 years for patients treated with SABR compared to 0.6 years for those who never received SABR. They suggested that the excellent local control achieved with SABR translated into a survival advantage. Navarria et $\mathrm{al}^{8}$ reported the SABR outcomes of 28 patients (51 lesions) with sarcoma lung metastases. Leiomyosarcoma and synovial sarcoma were the most common histologies. The SABR schemes included $30 \mathrm{~Gy}$ in one fraction, $60 \mathrm{~Gy}$ in three fractions, $60 \mathrm{~Gy}$ in eight fractions, and $48 \mathrm{~Gy}$ in four fractions. The actuarial 5-year local control rate was $96 \%$ and no severe toxicity was recorded. Baumann et $\mathrm{al}^{9}$ used SABR to treat 30 sarcoma patients with 39 pulmonary metastatic lesions. The median lesion size was 2.4 (range $0.5-8.1$ ) $\mathrm{cm}$, and the median dose was $50 \mathrm{~Gy}$ in four or five fractions. The 1- and 2-year local control rates were $94 \%$ and $86 \%$, respectively, with no grade $\geq 3$ toxicity. These studies have demonstrated that SABR is safe and effective for treating small numbers of small lung metastases (oligometastases) from soft tissue sarcoma.

The most important differential diagnosis of PLS is metastatic spread from primary extrapulmonary sarcoma. Sarcomas metastatic to the lung are far more frequent than PLS. ${ }^{1}$ PLS is histologically indistinguishable from metastatic lung sarcomas. ${ }^{3}$ Clinical and radiological examinations have found no evidence of an alternative primary source in the current case. As with soft tissue sarcomas of the extremities, a large size $(>5 \mathrm{~cm})$ and high grade of PLS are reportedly associated with worse patient survival., ${ }^{417}$ The small tumor targeted by SABR in the present patient may help explain the favorable outcome.

In conclusion, this case study demonstrates excellent tumor control with no severe toxicity following SABR for PLS. SABR may be a feasible option for not only metastatic lung sarcoma but also for PLS.

\section{Acknowledgment}

This work was supported by the Soonchunhyang University Research Fund. 


\section{Disclosure}

The author reports no conflicts of interest in this work.

\section{References}

1. Etienne-Mastroianni B, Falchero L, Chalabreysse L, et al. Primary sarcomas of the lung: a clinicopathologic study of 12 cases. Lung Cancer. 2002;38(3):283-289.

2. Litzky LA. Pulmonary sarcomatous tumors. Arch Pathol Lab Med. 2008;132(7):1104-1117.

3. Goncalves MJ, Mendes MM, Joao F, Lopes JM, Honavar M. Primary pleomorphic sarcoma of lung 11 year survival. Rev Port Pneumol. 2011;17(1):44-47.

4. Spraker MB, Bair E, Bair R, Connell PP, Mahmood U, Koshy M. An analysis of patient characteristics and clinical outcomes in primary pulmonary sarcoma. J Thorac Oncol. 2013;8(2):147-151.

5. Kim MJ, Yeo SG, Kim ES, Min CK, Se An P. Intensity-modulated stereotactic body radiotherapy for stage I non-small cell lung cancer. Oncol Lett. 2013;5(3):840-844.

6. Yeo SG, Kim MJ. Stereotactic body radiation therapy for the treatment of a post-chemotherapy remnant lung mass in extensive-stage small-cell lung cancer: a case report. Exp Ther Med. 2016;12(2):1185-1188.

7. Dhakal S, Corbin KS, Milano MT, et al. Stereotactic body radiotherapy for pulmonary metastases from soft-tissue sarcomas: excellent local lesion control and improved patient survival. Int J Radiat Oncol Biol Phys. 2012;82(2):940-945.

8. Navarria P, Ascolese AM, Cozzi L, et al. Stereotactic body radiation therapy for lung metastases from soft tissue sarcoma. Eur J Cancer. 2015;51(5):668-674.
9. Baumann BC, Nagda SN, Kolker JD, et al. Efficacy and safety of stereotactic body radiation therapy for the treatment of pulmonary metastases from sarcoma: a potential alternative to resection. $J$ Surg Oncol. 2016;114(1):65-69.

10. Zhang K, Deng H, Cagle PT. Utility of immunohistochemistry in the diagnosis of pleuropulmonary and mediastinal cancers: a review and update. Arch Pathol Lab Med. 2014;138(12):1611-1628.

11. Yeo SG, Kim ES. Efficient approach for determining four-dimensional computed tomography-based internal target volume in stereotactic radiotherapy of lung cancer. Radiat Oncol J. 2013;31(4):247-251.

12. Kim YS, Lee J, Park JI, Sung W, Lee SM, Kim GE. Volumetric modulated arc therapy for carotid sparing in the management of early glottic cancer. Radiat Oncol J. 2016;34(1):18-25.

13. Park S, Urm S, Cho H. Analysis of biologically equivalent dose of stereotactic body radiotherapy for primary and metastatic lung tumors. Cancer Res Treat. 2014;46(4):403-410.

14. Fowler JF. 21 years of biologically effective dose. Br J Radiol. 2010; 83(991):554-568.

15. Kim MS, Kim W, Park IH, et al. Radiobiological mechanisms of stereotactic body radiation therapy and stereotactic radiation surgery. Radiat Oncol J. 2015;33(4):265-275.

16. Timmerman R, Paulus R, Galvin J, et al. Stereotactic body radiation therapy for inoperable early stage lung cancer. JAMA. 2010;303(11): 1070-1076.

17. Sardenberg RA, Cangnaci Neto R, Cavalcanti F, Younes RN. Highgrade primary pulmonary leiomyosarcoma. Einstein (Sao Paulo). 2011;9(4):523-526.
OncoTargets and Therapy

\section{Publish your work in this journal}

OncoTargets and Therapy is an international, peer-reviewed, open access journal focusing on the pathological basis of all cancers, potential targets for therapy and treatment protocols employed to improve the management of cancer patients. The journal also focuses on the impact of management programs and new therapeutic agents and protocols on

\section{Dovepress}

patient perspectives such as quality of life, adherence and satisfaction. The manuscript management system is completely online and includes a very quick and fair peer-review system, which is all easy to use. Visit http://www.dovepress.com/testimonials.php to read real quotes from published authors. 